\title{
Anomalous U(1) as a Mediator of Supersymmetry Breaking
}

\author{
Gia Dvali and Alex Pomarol \\ Theory Division, CERN, CH-1211 Geneva 23, Switzerland
}

(Received 1 August 1996)

\begin{abstract}
We point out that an anomalous gauge U(1) symmetry is a natural candidate for being the mediator and messenger of supersymmetry breaking. It facilitates dynamical supersymmetry breaking even in the flat limit. Soft masses are induced by both gravity and the U(1) gauge interactions giving an unusual mass hierarchy in the sparticle spectrum which suppresses flavor violations. This scenario does not suffer from the Polonyi problem. [S0031-9007(96)01437-8]
\end{abstract}

PACS numbers: 12.60.Jv, 04.65.+e, 11.30.Na, 14.80.Ly

The origin of supersymmetry breaking remains an open question. More important, for phenomenological purposes, it is to know how the breaking of supersymmetry is transmitted to the ordinary particles. The most popular scenario arises in the context of supergravity. In these theories supersymmetry is assumed to be broken in some isolated hidden sector and transmitted to the observable sector by gravity [1]. These models, however, suffer from certain drawbacks. The degeneracy of the scalar quarks needed to avoid large flavor changing neutral currents (FCNC) is not usually guaranteed at low energies. Also the breaking of supersymmetry results in the nonflat limit leading to cosmological disasters (the Polonyi problem [2]).

In this Letter we will consider an alternative scenario. It is well known that extra U(1) factors normally appear in effective field theories arising from strings. One of these $U(1)$ is usually anomalous. The cancellation of its anomalies occurs by the Green-Schwarz mechanism [3] and requires that both hidden and observable fields transform nontrivially under this $\mathrm{U}(1)$. Thus this anomalous $\mathrm{U}(1)$ seems to be a natural new candidate for transmitting the supersymmetry breaking from the hidden to the observable sector. Here we will study this possibility.

Since the $\mathrm{U}(1)$ is anomalous, $\operatorname{Tr} \mathbf{Q} \neq 0$, a FayetIliopoulos term of $\mathcal{O}\left(M_{P}^{2}\right)$ is always generated [4]. This term facilitates the breaking of supersymmetry in the flat limit, avoiding the Polonyi problem. The scale of supersymmetry breaking can be smaller than $M_{P}$ and can originate dynamically. In the presence of gravity, realistic scalar and gaugino masses are induced in the observable sector. We find that the $D$-term contribution can be larger than the gravity mediated $F$-term contribution, resulting in a hierarchy of soft masses. This is a crucial difference with the conventional hidden sector scenarios in supergravity models. As we will show, our model can lead to a certain degree of squark degeneracy and suppressed FCNC. It also allows for an explanation of the observed quark mass hierarchy $\left(m_{t, b} \gg m_{u, d}, m_{c, s}\right)$ and predicts an inverse hierarchy for the squarks $\left(m_{\tilde{u}, \tilde{d}}^{2} \simeq\right.$ $\left.m_{\tilde{c}, \tilde{s}}^{2} \gg m_{\tilde{t}, \tilde{b}}^{2}\right)$.

Anomalous $\mathrm{U}(1)$ have been considered before to predict the weak mixing angle [5], fermion [6], or sfermion
[7] masses; in these previous analysis, however, the anomalous $U(1)$ does not play any role in the breaking of supersymmetry.

Supersymmetry breaking with an anomalous $U(1)$. Let us consider a pair of chiral superfields $\phi^{-}$and $\phi^{+}$ with charges equal to -1 and +1 , respectively, under a gauge $\mathrm{U}(1)$. We will assume that there are other positively charged fields $Q_{i}$ such that $\operatorname{Tr} \mathbf{Q}>0$ and the $\mathrm{U}(1)$ is anomalous. This results in the appearance of a Fayet-Iliopoulos term $\xi=\mathcal{O}\left(M_{P}^{2} \operatorname{Tr} \mathbf{Q}\right)$ [4]. In string theories the generated Fayet-Iliopoulos term can be calculated and is given by [8]

$$
\xi=\frac{g^{2} \operatorname{Tr} \mathbf{Q}}{192 \pi^{2}} M_{P}^{2} .
$$

The $D$-term contribution to the effective potential takes the form

$$
\frac{g^{2}}{2} D^{2}=\frac{g^{2}}{2}\left(\sum_{i} q_{i}\left|Q_{i}\right|^{2}+\left|\phi^{+}\right|^{2}-\left|\phi^{-}\right|^{2}+\xi\right)^{2},
$$

where $q_{i}$ is the $\mathrm{U}(1)$ charge of the field $Q_{i}$. If Eq. (2) is the only term in the potential, the vacuum expectation value (VEV) of $\phi^{-}$adjusts to compensate $\xi$, and supersymmetry will not be broken. However, according to the old observation by Fayet [9], this can lead to the spontaneous breakdown of the supersymmetry if the $\phi^{-}$field has a nonzero mass term in the superpotential:

$$
W=m \phi^{+} \phi^{-} .
$$

We will show below that such a mass term can, in fact, be generated dynamically. For the moment, let us consider it as a new input of the theory and look for its consequences. Minimization of the potential shows that the VEVs of the scalar components are

$$
\left\langle\phi^{+}\right\rangle=0, \quad\left\langle\phi^{-}\right\rangle^{2}=\xi-\frac{m^{2}}{g^{2}},
$$

and the VEVs of the $F$ and $D$ components are given by

$$
\left\langle F_{\phi^{+}}\right\rangle=m \sqrt{\xi-\frac{m^{2}}{g^{2}}}, \quad\left\langle F_{\phi^{-}}\right\rangle=0, \quad\langle D\rangle=\frac{m^{2}}{g^{2}} .
$$


The spectrum of the theory is the following: (1) The Goldstone boson $\operatorname{Im} \phi^{-}$is eaten up by the gauge field that gets a mass $g \sqrt{\xi-m^{2} / g^{2}}$ [10]; (2) its superpartner $\operatorname{Re} \phi^{-}$gets a mass $g \sqrt{\xi-m^{2} / g^{2}}$ from the $D$ term and becomes a member of the massive gauge superfield; (3) the complex scalar $\phi^{+}$gets a squared mass $2 m^{2}$; (4) one linear combination of the chiral fermions and the gaugino gets a Dirac mass $g \sqrt{\xi-m^{2} / 2 g^{2}}$, whereas the orthogonal combination is the massless Goldstino.

Let us now embed this model in a supergravity theory. It is easy to show that the broken global supersymmetry cannot be restored by the supergravity interactions. This is because an unbroken supergravity with vanishing vacuum energy implies $\langle W\rangle=0$ and therefore that all $\partial_{\phi} W$ and $D_{A}$ vanish too; this contradicts the initial assumption that supersymmetry was broken in the flat limit. Under supergravity, the VEVs of the fields will be shifted from Eqs. (4) and (5), but the relation

$$
\frac{\left\langle F^{2}\right\rangle}{\langle D\rangle} \sim \xi,
$$

will still hold.

The sparticle spectrum. - In a supergravity theory the supersymmetry breaking is communicated by gravity from the hidden sector $\left(\phi^{+}, \phi^{-}\right)$to the observable sector $\left(Q_{i}\right)$. The scalar masses receive contributions of order

$$
m_{Q}^{2} \simeq \frac{\left\langle F_{\phi^{+}}\right\rangle^{2}}{M_{P}^{2}} \simeq \frac{m^{2} \xi}{M_{P}^{2}} \simeq \varepsilon m^{2},
$$

where $\varepsilon \equiv \xi / M_{P}^{2}$ that in string theories takes the value $\varepsilon=g^{2} \operatorname{Tr} \mathbf{Q} / 192 \pi^{2}$. These contributions are, in principle, nonuniversal, since they depend on the Kähler potential [1]. The gaugino masses can arise from the operator

$$
\int d^{2} \theta \frac{\phi^{+} \phi^{-}}{M_{P}^{2}} W_{a} W_{a},
$$

where $W_{a}$ is the superfield that contains the gauge field strength of the standard model SU( $a)$ group, $a=1,2,3$. Thus gaugino masses are given by

$$
m_{\lambda} \simeq \frac{\left\langle F_{\phi^{+}} \phi^{-}\right\rangle}{M_{P}^{2}} \simeq \varepsilon m .
$$

Notice that the presence of the field $\phi^{-}$with a VEV of order $M_{P}$ is crucial to give acceptable gaugino masses from the operator equation (8). The absence of this field in other models in which supersymmetry is also broken in the flat limit leads to very light gauginos [11] (see, however, Ref. [12]). In string theories the operator equation (8) can only be induced at the one-loop level since only the dilaton couples to $W_{a} W_{a}$ at the tree level. Larger contributions to the gaugino masses, however, can arise from integrating out heavy states as we will show in the next section.

Since in our scenario $\langle D\rangle$ is different from zero, extra contributions to the scalar masses arise from the $D$ term for fields that transform under the anomalous U(1). From Eqs. (2) and (5), these are given by

$$
\Delta m_{Q_{i}}^{2}=q_{i} m^{2} .
$$

Notice that these contributions can be much larger than the $F$-term contributions Eq. (7) if $\varepsilon \ll 1$. Thus this scenario allows for a hierarchy of soft masses:

$$
\Delta m_{Q}^{2}>m_{Q}^{2}>m_{\lambda}^{2} .
$$

This is different from models in which the U(1) does not play any role in the breaking of supersymmetry. In those models the $D$-term contribution to the scalar masses is always of the same order as the $F$-term contribution [7]. The spectrum equations (7), (9), and (10) are a general feature of this hybrid scenario where the breaking of supersymmetry is transmitted by both gravity and $\mathrm{U}(1)$-gauge interactions and is due to the generic relation equation (6). This allows for a solution to the supersymmetric flavor problem, i.e., the required degeneracy between the first and second family squarks $\delta m_{Q}^{2} / m_{Q}^{2} \ll 1$. If these two families of squarks transform nontrivially under the $U(1)$, they receive the universal contribution of Eq. (10), which, for $\varepsilon \ll 1$, can be much larger than the nonuniversal contribution equation (7) and therefore

$$
\frac{\delta m_{Q}^{2}}{m_{Q}^{2}} \simeq \varepsilon \ll 1 .
$$

Decreasing $\varepsilon$ increases not only the degeneracy of the first two family squarks, but also increases their soft masses with respect to the other ones and then further suppresses the supersymmetric FCNC contributions. Obviously, $\varepsilon$ cannot be much smaller than 1, otherwise the gaugino masses obtained from (9) are too small. The best scenario that we envisage is to have the three quark families transforming under the $\mathrm{U}(1)$ as $\{1,1,0\}$, respectively [13]. For reasonable values of $\varepsilon=g^{2} \operatorname{Tr} \mathbf{Q} / 192 \pi^{2} \simeq 10^{-2}$, we get, for $m \simeq 5 \mathrm{TeV}$,

$$
m_{\lambda} \simeq 50 \mathrm{GeV}, \quad m_{Q_{3}} \simeq 500 \mathrm{GeV}, \quad m_{Q_{1,2}} \simeq 5 \mathrm{TeV} .
$$

This is a spectrum very similar to that in Ref. [14]. The FCNC are suppressed enough. Furthermore, this scenario provides a solution to the supersymmetric $C P$ problem [15]. This is because the first family of squarks are so heavy that their contribution to the electric dipole moment of the neutron is small, even if the $C P$-violating phases are of $\mathcal{O}(1)$. It is important to remark that the large masses $m_{Q_{1,2}}$ do not lead to a naturalness problem, since $Q_{1,2}$ are almost decoupled from the Higgs boson $[14,16]$. This is because $\operatorname{Tr}[\mathbf{Q Y}]=0$ (where $\mathbf{Y}$ is the hypercharge generator) and the soft masses of the Higgs boson are only affected by $m_{Q_{1,2}}$ at the two-loop level (neglecting the small Yukawas) when they evolve from $M_{P}$ to the weak scale $[14,16]$. 
The above anomalous $\mathrm{U}(1)$ could also play a role in explaining the fermion masses in the same spirit as in Ref. [6]. Here, however, we are constrained to have the first two families with equal $\mathrm{U}(1)$ charges (in order to avoid too large FCNC) [13]. Although a complete model will not be attempted in this Letter, it is interesting to note that if, as we mentioned above, the Higgs boson and the third family are neutral under this $U(1)$ but the first and second ones are charged, a tree-level mass is only allowed for the third family, explaining why the top and bottom masses are much larger than the others. This scenario relates the mass hierarchy of the quarks to that in Eq. (13) for the squarks.

It is worthwhile to point out that, contrary to most of the flavor models, our scenario allows for gauging extra flavor symmetries, since the universal contribution equation (10) dominates over any other nonuniversal $D$-term contribution.

A scenario of dynamical supersymmetry breaking. - Up to now we have assumed that $m \sim 1 \mathrm{TeV}$ is just a new scale in the model. In this section we will show that this scale can be generated dynamically. We only need a gauge group that at some intermediate scale $\Lambda$ becomes strongly interacting and leads to a field condensation.

The simplest example is an $\mathrm{SU}(2)$ group with two doublets $\Phi$ and $\Phi$, neutral under the anomalous U(1) [17]. At energies below the scale $\Lambda$, the low-energy effective theory can be described in terms of the gauge-invariant quantity $X \equiv \Phi \bar{\Phi}[11]$. The superpotential is given by

$$
W=\lambda \frac{X}{M_{P}} \phi^{+} \phi^{-}+\frac{\Lambda^{5}}{X},
$$

where the first term has been assumed to be present in the classical theory; the second term is generated nonperturbatively by instantons [11]. If no Fayet-Iliopoulos term is present in the theory, the vacuum has a run-away behavior, $X \rightarrow \infty$ with $\phi^{+}, \phi^{-} \rightarrow 0$. However, when the U(1) $D$ term of Eq. (2) is considered, the field $\phi^{-}$is forced to get a VEV and drives $X$ to a value around $\Lambda$. This generates the effective scale $m=\lambda\langle X\rangle / M_{P}$ and the breaking of supersymmetry. The only difference with respect to the model of Eq. (3) is that $\phi^{+}$now gets a VEV of order $\sqrt{\xi}$ and then $\left\langle F_{\phi^{-}}\right\rangle \sim m \sqrt{\xi}$. A new contribution to the gaugino masses can now arises from the operator

$$
\frac{1}{16 \pi^{2}} \int d^{2} \theta \frac{\phi^{-}}{\sqrt{\xi}} W_{a} W_{a},
$$

which can be induced if extra heavy matter fields (transforming under the standard model group) are present and get their masses from couplings to $\phi^{-}$. It can be shown that these couplings do not modify the supersymmetrybroken vacuum. Although the operator equation (15) is suppressed by a one-loop factor, it is enhanced with respect to the gravity-induced operators since $\sqrt{\xi}<M_{P}$. Equation (15) generates a mass term for the gauginos given by

$$
m_{\lambda} \simeq \frac{1}{16 \pi^{2}} \frac{\left\langle F_{\phi^{-}}\right\rangle}{\sqrt{\xi}} \simeq \frac{m}{16 \pi^{2}}
$$

that can be as large as Eq. (9).

The simplicity of this dynamical model resides in the fact that the strongly interacting gauge group is only needed for generating the small scale $m$ and not for breaking the supersymmetry by itself as in Ref. [11]. Here it is the Fayet-Iliopoulos term that plays the new and crucial role of triggering the breaking of supersymmetry.

The Polonyi problem. - Perhaps the main cosmological difficulty of the supergravity models with a conventional hidden sector is the Polonyi problem [2]. This arises because models in which supersymmetry gets restored in the flat limit predict light $\mathcal{O}\left(m_{3 / 2}\right)$ scalar particles with VEVs of $\mathcal{O}\left(M_{P}\right)$, with an extremely flat potential and $1 / M_{P}$ suppressed interactions. In the early Universe these fields are expected to sit far away from their present (zero-energy) vacua. The reason is that in the early Universe (during inflation or in the heat bath) these flat directions get large soft masses equal to $\alpha H^{2}$, where $H$ is the Hubble parameter and $\alpha$ is a number of order 1 that depends on the details of the cosmological scenario [18]. For particles with nonzero VEVs this leads, almost for sure, to a classical displacement from the present vacuum at the early times $\left(\Delta \sim M_{P}\right)$ and to the subsequent coherent oscillations around the true minimum after inflation. The amplitude and consequently the energy stored in the oscillations is determined by the initial deviation and will overclose the Universe if the displacement is larger than $\sim 10^{-9} M_{P}$ [2]. For $\alpha>0$ the displacement is generically given by the value of the present VEV, whereas for $\alpha<0$ it can be much larger. Therefore a light decoupled scalar with a VEV larger than $10^{-9} M_{P}$ is problematic, whereas scalars with smaller VEVs (at present) can be diluted by inflation. Now it is clear why the Polonyi problem can be overcome in theories with flat space supersymmetry breaking. Such theories do not necessarily require scalars with large VEVs and vanishing mass in the globally supersymmetric limit. In our models, the field that gets a VEV of order $M_{P}$ is heavy; it is eaten up by the massive $\mathrm{U}(1)$-gauge superfield.

We conclude with the following remarks.

(i) We pointed out that an anomalous gauge U(1) symmetry is a natural candidate for being the mediator and messenger of supersymmetry breaking. It allows for simple models of dynamical supersymmetry breaking in the flat limit.

(ii) These models can be embedded in a supergravity theory and generate realistic scalar and gaugino soft masses. The supersymmetry breaking is communicated by gravity and the gauge $\mathrm{U}(1)$. This hybrid scenario allows for a solution to the supersymmetric flavor and $C P$ problem since the first and second family of squarks are heavy. 
The resulting phenomenology is very different from that of the usual models with universal soft masses [14].

(iii) Since supersymmetry is broken in the flat limit, there is no Polonyi problem. All the hidden sector fields are either very massive or get VEV below the Planck scale.

It is a pleasure to thank Gian Giudice, Amit Giveon, Luis Ibáñez, Fernando Quevedo, and Misha Shifman for very useful discussions.

Note added.-After submitting this paper, we learned about a related work by P. Binétruy and E. Dudas, Report No. hep-th 9607172. We thank E. Dudas for comments.

[1] See, for example, H. P. Nilles, Phys. Rep. 110, 1 (1984).

[2] G. D. Coughlan, W. Fischler, E. W. Kolb, S. Raby, and G. G. Ross, Phys. Lett. B 131, 59 (1983); J. Ellis, D. V. Nanopoulos, and M. Quirós, Phys. Lett. B 147, 176 (1986); B. de Carlos, J. A. Casas, F. Quevedo, and E. Roulet, Phys. Lett. B 318, 447 (1993); T. Banks, D. Kaplan, and A. Nelson, Phys. Rev. D 49, 779 (1994).

[3] M. Green and J. Schwarz, Phys. Lett. B 149, 117 (1984).

[4] E. Witten, Nucl. Phys. B188, 513 (1981); W. Fischler et al., Phys. Rev. Lett. 47, 657 (1981).

[5] L. Ibáñez, Phys. Lett. B 303, 55 (1993).

[6] See, for example, L. Ibáñez and G. G. Ross, Phys. Lett. B 332, 100 (1994).

[7] H. Nakano, Report No. hep-th/9404033; Y. Kawamura and T. Kobayashi, Phys. Lett. B 375, 141 (1996); E. Dudas, S. Pokorski, and C. A. Savoy, Phys. Lett. B 369, 255 (1996); E. Dudas, C. Grojean, S. Pokorski, and C. A. Savoy, Report No. hep-ph/9606383.

[8] M. Dine,N. Seiberg, and E. Witten, Nucl. Phys. B289, 585 (1987); J. Atick, L. Dixon, and A. Sen, Nucl. Phys. B292,
109 (1987); M. Dine, I. Ichinose, and N. Seiberg, Nucl. Phys. B293, 253 (1987).

[9] P. Fayet, Nucl. Phys. B90, 104 (1975).

[10] In string theories the eaten up Goldstone is a superposition of $\operatorname{Im} \phi^{-}$and the model-independent axion (partner of the dilaton) [8].

[11] I. Affleck, M. Dine, and N. Seiberg, Nucl. Phys. B256, 557 (1985); A. I. Vainshtein, V. I. Zakharov, and M. A. Shifman, Sov. Phys. Usp. 28, 709 (1985).

[12] A. Nelson, Phys. Lett. B 369, 277 (1996); K.-I. Izawa and T. Yanagida, Prog. Theor. Phys. 95, 829 (1996); K. Intriligator and S. Thomas, Report No. hep-th/9603158.

[13] One can consider other possibilities such as equally charging only the left-handed squarks of the first two families. This can be enough to avoid the FCNC constraints coming from the real part of the $K^{0}-\bar{K}^{0}$ mass mixing if the corresponding right-handed squarks are heavier than $\sim 5 \mathrm{TeV}$.

[14] A. Pomarol and D. Tommasini, Nucl. Phys. B466, 3 (1996).

[15] W. Buchmüller and D. Wyler, Phys. Lett. B 121, 321 (1983); J. Polchinski and M. B. Wise, Phys. Lett. B 125, 393 (1983); Y. Kizukuri and N. Oshimo, Phys. Rev. D 46, 3025 (1992).

[16] S. Dimopoulos and G. Giudice, Phys. Lett. B 357, 573 (1995).

[17] Notice that anomaly cancellation by the Green-Schwarz mechanism requires fields transforming under both groups, U(1) and the strong SU(2). Such extra fields should be added in a complete model, but this would not be attempted here. It can be shown that the fields can be added without altering our conclusions.

[18] G. Dvali, Report No. hep-ph/9503259; Phys. Lett. B 355, 78 (1995); M. Dine, L. Randall, and S. Thomas, Phys. Rev. Lett. 75, 398 (1995). 